\title{
Peertechz
}

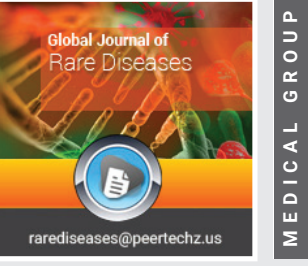

\section{Time to pay attention: Elder abuse in the COVID-19 Pandemic Era}

\author{
Nader Aghakhani ${ }^{1}$, Kazhal Mobaraki ${ }^{2}$, Marzieh Hossini ${ }^{3}$ and \\ Jamal Ahmadzadeh ${ }^{2 *}$
}

Check for updates

Received: 03 December, 2020

Accepted: 30 June, 2021

Published: 01 July, 2021

*Corresponding author: Jamal Ahmadzadeh, MSc in Epidemiology, Social Determinants of Health Research Center, Urmia University of Medical Sciences, Urmia, Iran, Tel: +98-9143444924; Fax: +98-4432240642; E-mail: Ahmadzadeh.j@umsu.ac.ir

Keywords: COVID-19; Personal protective measures; Older adults; Pandemic; Public health

https://www.peertechzpublications.com

${ }^{1}$ Associate Professor of Nursing, Patient Safety Research Center, Urmia University of Medical

Sciences, Urmia, Iran

${ }^{2} \mathrm{MSc}$ in Epidemiology, Social Determinants of Health Research Center, Urmia University of Medical

Sciences, Urmia, Iran

${ }^{3} \mathrm{MSc}$ in Clinical Psychology, Islamic Azad University of Urmia, Urmia, Iran

\begin{abstract}
COVID 19 pandemic has presented an opportunity for health care providers to expand their understanding of risks of using neglectful or abusive behavior to the elder during this time, and consider incorporating studies related to this challenge. Individuals experiencing caregiving concern and stress about whether their loved ones' needs will be met should be considered. They should be more open to share their uncomfortable experiences. Being aware of the problems of older adults and their caregivers is critical to helping them thrive and survive during this pandemic. Addressing increased risks, attending to mental health needs, and connecting older adults to caregiving and providing financial resources can all help the old patients and their loved ones to avoid violent and abusive situations and be safer.
\end{abstract}

Since the first case of the COVID-19 pandemic was diagnosed, a new policy measures have been enacted to prevent the public from its growing transmission. Social distancing, the shuttering of non-essential businesses and school closures have led to social, personal and economic restriction. It has been publicized that older persons are at higher risk of death from COVID-19 illness and healthcare staffs should access needed supplies or care for them and be aware that they may be particularly vulnerable to financial hardship, social isolation and anxiety after emerging COVID-19. Unfortunately, they may be at increased risk of abuse as an important public health problem [1] varies from incidents of family violence to financial scams that can lead to serious long-term psychological consequences or physical injuries due to vulnerability often resulting from pre-existing and age-related health conditions. Even in the best of times, prevalence rates of it may likely to be rarely detected or underestimated, because they are afraid to report to family or to the authorities [2].

Risk factors of abuse can be divided to individual, relationship, community, and socio-cultural dimensions.
- Risks at the individual dimension include poor physical and mental health of the old person, and mental disorders and substance or alcohol abuse in the abuser. A shared living situation and gender of victim may increase the risk of abuse.

- Risks at the relationship dimension include an abuser's dependency on the older person (often financial) that can increase the risk of abuse.

- Risk at community dimension include social isolation of older persons and their caregivers and the ensuing lack of social support. Many elderly persons are isolated because of loss of mental or physical capacity, or through the loss of family members and friends.

- Risk at socio-cultural dimension include lack of funds to pay for care, ageist stereotypes erosion of the bonds between generations of a family and systems affecting the distribution of material goods and power within families [3]. 
On the other hand, family and friends as caregivers of older adults are now facing financial strains which can increase their risk of being neglectful or abusive to their older family members. In addition, many of them are experiencing increased time caring for children home from closed schools or for other ill family members that have increased time demands, financial stressors, and worries about their care recipients. All of these factors can inadvertently lead to neglect of older adults.

In addition, many caregivers want to use virtual technologies to connect with their old relatives with low ability to have real contact. While some older persons with limited resources cannot have high-speed internet, smart phone or computer to use that may have health and personal consequences or lead to neglect of care [4].

In the face of the challenges caused by the current COVID-19 crisis, the healthcare providers can do video or telephonic visits with older adult patients or visit them in their environment for signs of unsafe situations, being cared for by others, and caring for themselves. They should provide support for caregivers who may not typically attend clinic visits, especially for the most vulnerable older adult patients (e.g., those with deterioration in behaviour, thinking, memory and the ability to perform their everyday activities) and should assess caregiver stress, ability to access necessary supplies and maintain previous levels of caregiving and then provide problem-solving strategies, brief counseling, and appropriate referrals.

Local area agencies can be another resource for providing a range of services to meet the needs of older persons, including personal care services, home-delivered meals, chronic disease and health promotion management, social engagement, and transportation [5].

Encouraging older patients to forge bonds may be possible during this pandemic. They can be encouraged to connect to volunteers who can pick up needed groceries or neighboring families who may help check on their wellbeing and to local agencies or charities that can donate supplies to them, which may help decrease isolation, fill in gaps, and reduce unmet needs [6].

\section{Conclusion}

In conclude, COVID 19 pandemic has presented an opportunity for health care providers to expand their understanding of risks of using neglectful or abusive behavior to the elder during this time, and consider incorporating studies related to this challenge. Individuals experiencing caregiving concern and stress about whether their loved ones' needs will be met should be considered. They should be more open to share their uncomfortable experiences.

Being aware of the problems of older adults and their caregivers is critical to helping them thrive and survive during this pandemic. Addressing increased risks, attending to mental health needs, and connecting older adults to caregiving and providing financial resources can all help the old patients and their loved ones to avoid violent and abusive situations and be safer.

\section{References}

1. Wu Z, McGoogan JM (2020) Characteristics of and Important Lessons from the Coronavirus Disease 2019 (COVID-19) Outbreak in China: Summary of a Report of 72314 Cases from the Chinese Center for Disease Control and Prevention. JAMA 323: 1239-1242. Link: https://bit.ly/3h4fDVT

2. Dong $E$, Du H, Gardner L (2020) An interactive web-based dashboard to track COVID-19 in real time. Lancet Infect Dis 20: 533-534. Link: https://bit.ly/3ycM36e

3. WHO (2021) Elder abuse. Link: https://bit.ly/3xmJZsF

4. Duggan $P$, Weiner $R(2020)$ Gun and ammunition sales rise amid pandemic fears. Washington Post. Link https://wapo.st/3w3Ev4x

5. Brewster AL, Wilson TL, Kunkel SR, Markwood S, Shah TB (2020) To Support Older Adults Amidst The COVID-19 Pandemic, Look to Area Agencies On Aging. Link: https://bit.ly/3xb0RSL

6. Makaroun L, Bachrach R, Rosland A (2020) Elder Abuse in the time of COVID-19 - Increased Risks for Older Adults and Their Caregivers. Am J Geriatr Psychiatry 28: 876-880. Link: https://bit.ly/3649oLx

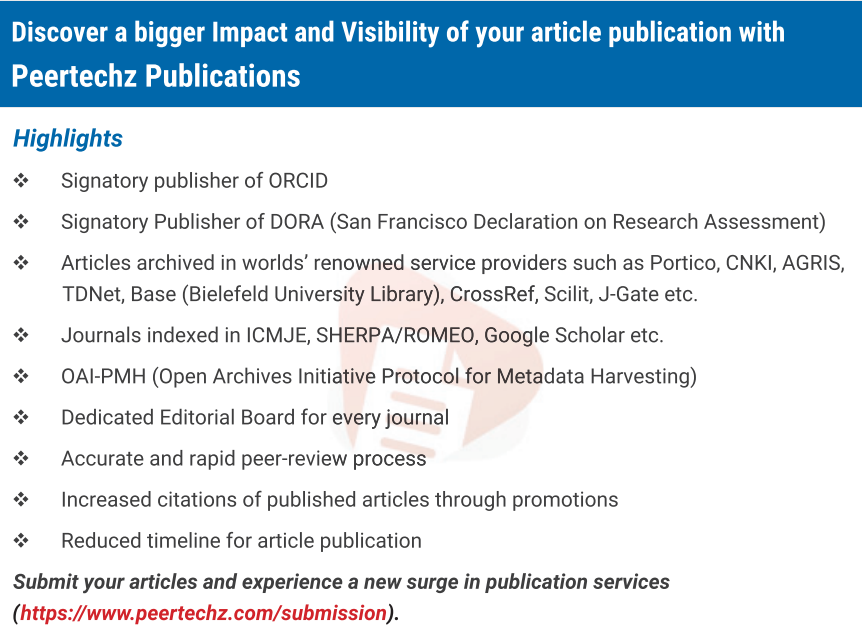
Peertechz journals wishes everlasting success in your every endeavours.

Copyright: @ 2021 Aghakhani N, et al. This is an open-access article distributed under the terms of the Creative Commons Attribution License, which permits unrestricted use, distribution, and reproduction in any medium, provided the original author and source are credited.

Citation: Aghakhani N, Mobaraki K, Hossini M, Ahmadzadeh J (2021) Time to pay attention: Elder abuse in the CoVID-19 Pandemic Era. Glob J Rare Dis 6(1): 004005. DOI: https://dx.doi.org/10.17352/2640-7876.000030 